\title{
Epidural motor cortex stimulation with functional imaging guidance
}

\author{
Alon Y. Mogilner, M.D., Ph.D., ANd Ali R. Rezai, M.D. \\ Department of Neurosurgery, New York Medical College, Valhalla, and New York, New York; and \\ Department of Neurosurgery, Cleveland Clinic Foundation, Cleveland, Ohio
}

\begin{abstract}
Chronic epidural motor cortex stimulation (MCS) has been shown to have promise in the treatment of patients with refractory deafferentation pain. Precise placement of the electrode over the motor cortex region corresponding to the area of pain is essential for the success of this procedure. Whereas standard anatomical landmarks have been used in the past in conjunction with image guidance, the use of functional brain imaging can be beneficial in the precise surgical planning. The authors report the use of functional imaging-guided frameless stereotactic surgery for epidural MCS. Five patients underwent MCS in which functional imaging guidance was used. Prior to surgery, patients underwent magnetic resonance (MR) imaging with skin fiducial markers placed on standard anatomical reference prints, followed by magnetoencephalography (MEG) mapping of the sensory and motor cortices. In two patients, functional MR imaging was also performed using a motor task paradigm. The functional imaging data were integrated into a frameless stereotactic database by using a three-dimensional coregistration algorithm. Subsequently, a frameless stereotactic craniotomy was performed using the integrated anatomical and functional imaging data for surgical planning. Intraoperative somatosensory evoked potentials (SSEPs) and direct stimulation were used to confirm the target and final placement of the electrode.

Direct stimulation and SSEPs performed intraoperatively confirmed the accuracy of the functional imaging data. Trial periods of stimulation successfully reduced pain in three of the five patients who then underwent permanent internal placement of the system. At a mean 6-month follow up, these patients reported an average reduction in pain of 55\% on a visual analog scale. The integration of functional and anatomical imaging data allows for precise and efficient surgical planning and may reduce the time necessary for intraoperative physiological verification.
\end{abstract}

\section{KEY WORDS • motor cortex stimulation • frameless stereotaxis • magnetoencephalography • functional magnetic resonance imaging}

The use of epidural MCS for the treatment of refractory deafferentation pain was first described by Tsubokawa and colleagues ${ }^{14,15}$ in 1991. Since their initial reports, other groups have reported procedure-related success in treating deafferentation pain syndromes of varying causes, with success rates ranging from 50 to $80 \%$ for both central and trigeminal neuropathic pain. ${ }^{1,6,8}$ This procedure, in which minimal rates of morbidity have been demonstrated, is gaining increasing acceptance.

Precise intraoperative placement of the stimulating electrode over the motor cortex region corresponding to the painful body part is essential to successful outcome. Although most groups have reported intraoperative electrophysiological confirmation of motor cortex by using SSEP and/or EMG monitoring, initial localization techniques vary. The use of surface anatomical landmarks, scalp SSEPs, and computerized tomography and MR imaging-guided systems have all been reported. ${ }^{1,3,7,8,13}$

\footnotetext{
Abbreviations used in this paper: $\mathrm{EMG}=$ electromyography; $\mathrm{fMR}=$ functional magnetic resonance; $\mathrm{MCS}=$ motor cortex stimulation; $\mathrm{MEG}=$ magnetoencephalography; SSEP = somatosensory evoked potential.
}

Functional imaging information, such as that obtained using MEG, fMR imaging, and optical imaging, is now routinely integrated into many neurosurgical procedures, including tumor/arteriovenous malformation resection and epilepsy surgery. Sole reliance on anatomical criteria for determination of eloquent cortex has been shown to have limitations, and the integration of such functional imaging data is a useful adjunct in preoperative planning and risk assessment as well as for intraoperative guidance. ${ }^{4,10-14}$ We report our experience using functional imaging guidance with MEG as well as fMR imaging in patients undergoing MCS for the treatment of refractory pain.

\section{CLINICAL MATERIAL AND METHODS}

Five patients underwent functional imaging-guided epidural MCS. Indications for the procedure included atypical facial pain in three patients, brachial plexus avulsion in one, and post-spinal cord injury lower-extremity pain in one. In all cases pain was refractory to standard medical therapy, and the patients underwent comprehensive neurological and psychological evaluation prior to being considered for surgery. 
The day prior to surgery, patients underwent MR imaging with skin fiducial markers placed on standard anatomical reference points used by the MEG system-the nasion and the left and right preauricular points. Additional fiducial markers were placed as indicated for the image guidance system. An fMR imaging motor activation paradigm involving movement of the affected body part was performed in two patients. Following the MR imaging session, the patients underwent MEG mapping of the sensory and motor cortices, performed on a 148-channel Magnes system (WH2500; 4-D Neuroimaging, San Diego, CA; Fig. 1). Somatosensory mapping, performed in all patients, consisted of recording and localizing evoked responses to tactile stimulation of the affected body part. Motor mapping, performed for the upper extremity, consisted of paced repetitive finger tapping movements, with the evoked responses triggered by EMG activity. In both sensory and motor mapping, evoked responses were averaged 250 times and filtered from 3 to $40 \mathrm{~Hz}$. Using a single dipole model, activity was then localized onto the corresponding MR image by using standard MEG software.

Following functional image acquisition, the data were transferred over the network to the neurosurgical imageguided system (Cygnus PFS; Compass International, Rochester, MN). The necessary coordinate transformation to integrate the functional imaging data into the surgical database was performed with in-house software, allowing the coordinates for the sensory and motor cortices of the affected body part to be displayed within the standard surgical planning software (Fig. 2 upper). ${ }^{10}$

Patients underwent an awake craniotomy in which frameless stereotaxis was used. Intravenous sedation with Diprivan and midazolam was used, and the skin was anesthetized using a mixture of $1 \%$ lidocaine $/ 0.25 \%$ bupivicaine. Using the image guidance system, the fiducial markers were registered to the scalp (Fig. 2 lower), the sensorimotor region was localized on the skin based on

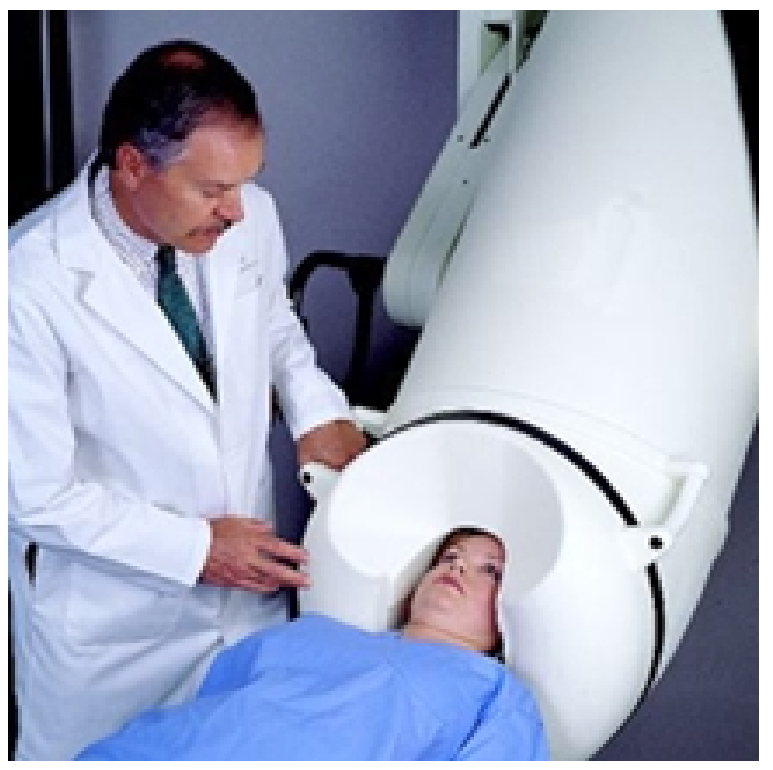

Fig. 1. Photograph showing the 148-channel whole-head neuromagnetometer system.

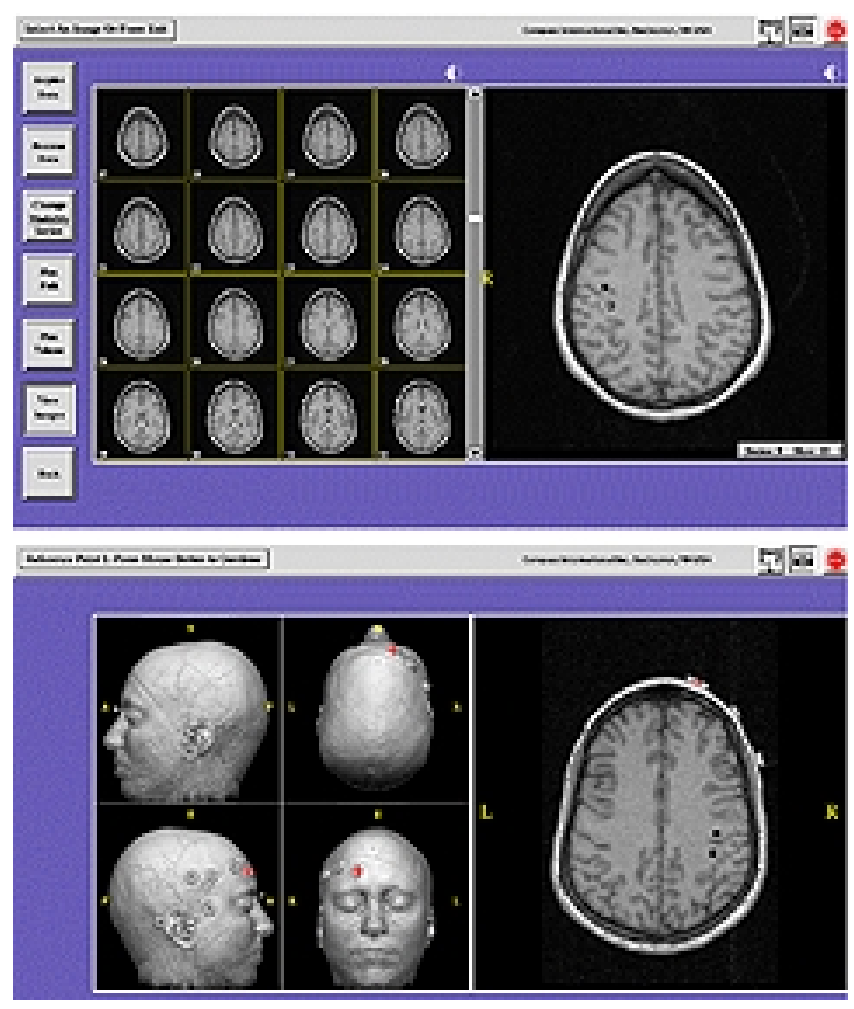

Fig. 2. Integration of MEG data into the frameless database. The locations of the MEG current dipoles representing the motor cortex (circles) and the sensory cortex (squares) are shown on an axial slice in the imaging database prior to (upper) and during (lower) the process of image registration.

the integrated functional and anatomical imaging data (Fig. 3 upper), and the approximate location of the central sulcus was outlined (Fig. 3 lower). A curvilinear incision was made, centered over the motor cortex, and a rectangular craniotomy was made, exposing the dura. Epidural SSEPs recorded with an eight-contact electrode strip in response to median and trigeminal nerve stimulation were used to locate the site of phase reversal corresponding to the central sulcus. Once the region of the central sulcus was identified, a standard quadripolar laminectomy-type electrode (Resume II, model 3587A; Medtronic, Minneapolis, MN) was placed over the dura, and direct stimulation was provided through the electrode to locate the cortical representation of the painful body part. Visual inspection of the facial and extremity muscles as well as EMG recordings were used to aid in localization. Once the appropriate location was determined, the electrode was sutured to the dura overlying the motor cortex (Fig. 4) and connected to an extension lead for the externalized trial period. Prophylactic anticonvulsant agents were given during the trial period.

Patients then underwent an in-hospital trial period of 2 to 5 days to determine if stimulation, performed at approximately two thirds the voltage threshold necessary for motor contractions, would provide significant pain relief, defined as greater than $50 \%$ pain reduction on a visual analog scale. If the trial period was successful, the system was placed internally and connected to a subcutaneous pulse generator (Itrel 3; Medtronic). 


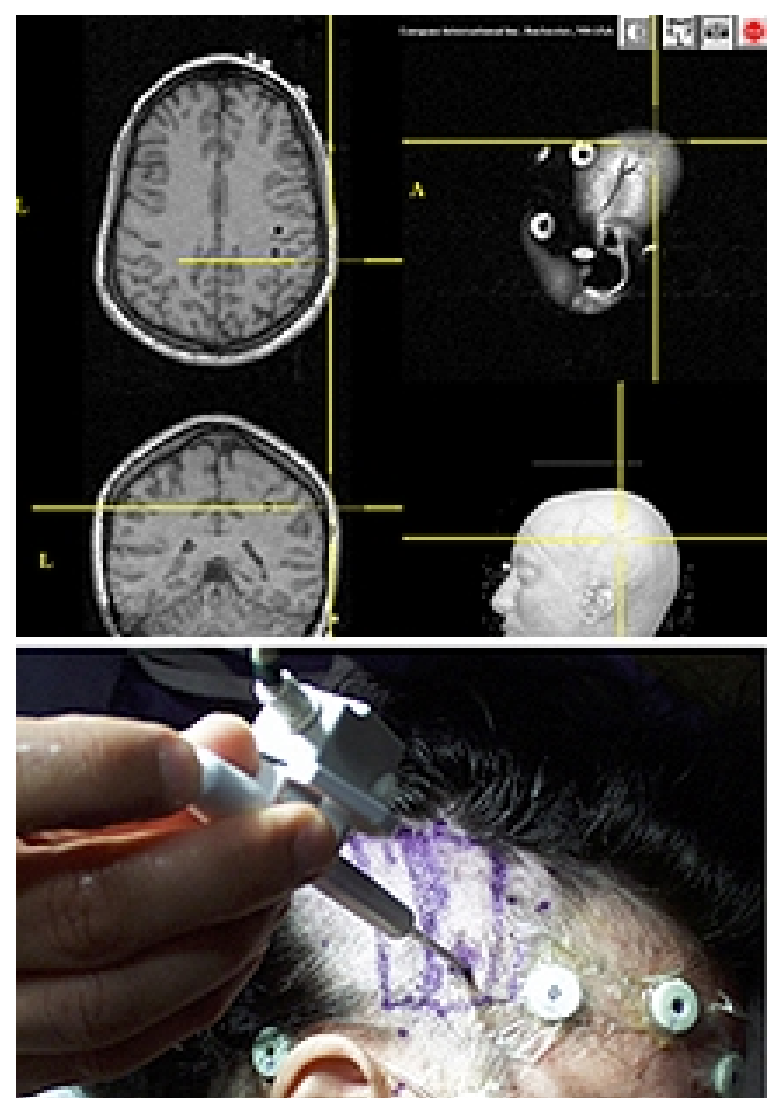

Fig. 3. Upper: Interactive use of the image guidance system with integrated functional imaging information to determine the region of the motor cortex. The yellow crosshairs represent the position of the pointing device on the skin relative to the MR imaging and MEG data. Lower: Photograph showing the approximate location of the pre- and postcentral gyri and the central sulcus as determined by the frameless stereotactic system. Using the pointing device, the skin incision and craniotomy can be planned to include the appropriate region(s) of sensorimotor cortex.

\section{RESULTS}

In conjunction with the image guidance system, the functional imaging data were used to plan the skin inci- sion and craniotomy and to guide initial placement of electrodes. In all cases, there was excellent correspondence between the location of the central sulcus and motor cortex as determined by functional imaging and that determined by intraoperative physiological inspection. Trial periods were successful in three patients in whom the system was then placed internally. At last follow-up examination (mean 6 months), visual analog scale scores indicated a mean pain reduction with stimulation of $55 \%$ in these patients. Stimulation parameters were a frequency of $110 \mathrm{~Hz}$, pulse width of $210 \mu \mathrm{sec}$, and an amplitude range of 2 to $8 \mathrm{~V}$, at approximately two thirds the threshold for motor contraction. Stimulation was performed from five to 10 times per day, with the duration of each session ranging from approximately 30 minutes to 2 hours.

\section{DISCUSSION}

Accurate localization of motor cortex and precise electrode placement are essential for maximal clinical efficacy of the procedure. Although MCS is a relatively new technique, a number of technical procedural variations have already been described. In the initial studies by Tsubokawa and colleagues ${ }^{14}$ and subsequently by Meyerson, et al., ${ }^{5}$ the authors reported using a single burr hole centered over the motor cortex. The location of the burr hole was determined by standard anatomical landmarks and skin-surface SSEPs, and the electrode was placed through the burr hole over the approximate location of the motor cortex..$^{5,15}$ In later reports by Ebel, et al., ${ }^{2}$ and Peyron, et al., ${ }^{9}$ the authors suggested that a craniotomy should be used rather than a burr hole, allowing for more extensive mapping of the rolandic region. Further refinement of the technique with the introduction of anatomical image guidance, as described by Nguyen and colleagues, ${ }^{7,8}$ has been associated with improved long-term results. Although not all neurosurgeons have used image guidance, ${ }^{1}$ all perform intra- or postoperative direct functional mapping (SSEPs or direct stimulation) to guide placement of the stimulating electrode. Although functional imaging data such as that obtained using fMR imaging and MEG will never replace intraoperative physiological localization, the integration of such information into an image guidance system can serve as a useful adjunct and may allow for more

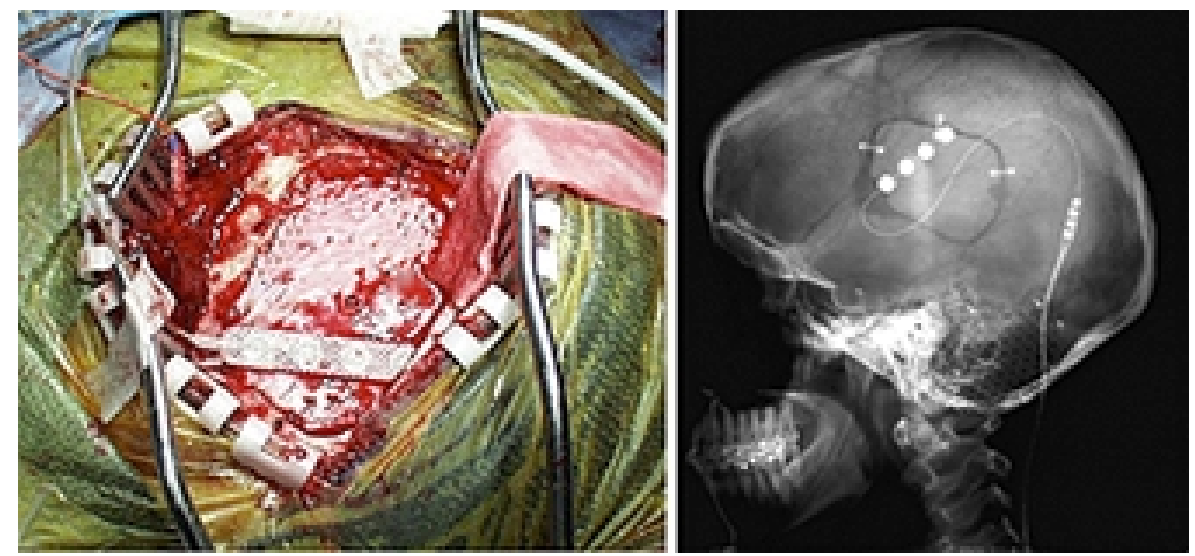

Fig. 4. Left: Photograph showing the permanent electrode sutured to the dura. Right: Lateral radiograph demonstrating the craniotomy and final electrode placement. 
efficient planning and thus reduce the time necessary for the procedure. To our knowledge there have been no prior reports in which MEG guidance was used to perform this procedure, and there is one report ${ }^{13}$ in the literature in which the authors used fMR imaging for MCS.

Functional imaging is being increasingly used in neurosurgical procedures. Magnetoencephalography is a noninvasive functional imaging modality that is used to record and localize the magnetic activity generated by the nervous system as a direct consequence of neuronal activity. Functional MR imaging, which measures blood oxygenation and/or flow, is an indirect measure of neuronal activation. Whereas both fMR imaging and MEG provide excellent spatial resolution, MEG provides superior temporal resolution, on the order of milliseconds. Both of these imaging modalities can be routinely integrated into frame-based and frameless image guidance systems. This has been used to help guide resection of tumors and vascular malformations associated with eloquent cortex. ${ }^{12}$ Similarly, this combination of functional and anatomical information can be used to guide MCS procedures. We now routinely perform $\mathrm{MEG}$ and fMR imaging in our patients and integrate the information into our image guidance systems to assist in preoperative surgical planning and in intraoperative localization. The integration of preoperative functional imaging data into a frameless stereotactic database provides an efficient, precise, and reliable method of performing MCS surgery.

\section{References}

1. Carroll D, Joint C, Maartens N, et al: Motor cortex stimulation for chronic neuropathic pain: a preliminary study of 10 cases. Pain 84:431-437, 2000

2. Ebel H, Rust D, Tronnier V, et al: Chronic precentral stimulation in trigeminal neuropathic pain. Acta Neurochir 138: 1300-1306, 1996

3. Herregodts P, Stadnik T, De Ridder F, et al: Cortical stimulation for central neuropathic pain: 3-D surface MRI for easy determination of the motor cortex. Acta Neurochir Suppl 64: 132-135, 1995

4. Hund M, Rezai AR, Kronberg E, et al: Magnetoencephalo- graphic mapping: basic of a new functional risk profile in the selection of patients with cortical brain lesions. Neurosurgery 40:936-943, 1997

5. Meyerson BA, Lindblom U, Linderoth B, et al: Motor cortex stimulation as treatment of trigeminal neuropathic pain. Acta Neurochir Suppl 58:150-153, 1993

6. Nguyen JP, Keravel Y, Feve A, et al: Treatment of deafferentation pain by chronic stimulation of the motor cortex: report of a series of 20 cases. Acta Neurochir Suppl 68:54-60, 1997

7. Nguyen JP, Lefaucheur JP, Decq P, et al: Chronic motor cortex stimulation in the treatment of central and neuropathic pain. Correlations between clinical, electrophysiological and anatomical data. Pain 82:245-251, 1999

8. Nguyen JP, Lefaucher JP, Le Guerinel C, et al: Motor cortex stimulation in the treatment of central and neuropathic pain. Arch Med Res 31:263-265, 2000

9. Peyron R, Garcia-Larrea L, Deiber MP, et al: Electrical stimulation of precentral cortical area in the treatment of central pain: electrophysiological and PET study. Pain 62:275-286, 1995

10. Rezai AR, Hund M, Kronberg E, et al: The interactive use of magnetoencephalography in stereotactic image-guided neurosurgery. Neurosurgery 39:92-102., 1996

11. Rezai AR, Hund M, Kronberg E, et al: Introduction of magnetoencephalography to stereotactic techniques. Stereotact Funct Neurosurg 65:37-41, 1995

12. Rezai AR, Mogilner AY, Cappell J, et al: Integration of functional brain mapping in image-guided neurosurgery. Acta Neurochir Suppl 68:85-89, 1997

13. Roux FE, Ibarrola D, Lazorthes Y, et al: Chronic motor cortex stimulation for phantom limb pain: a functional magnetic resonance imaging study: technical case report. Neurosurgery 48: 681-688, 2001

14. Tsubokawa T, Katayama Y, Yamamoto T, et al: Chronic motor cortex stimulation in patients with thalamic pain. J Neurosurg 78:393-401, 1993

15. Tsubokawa T, Katayama Y, Yamamoto T, et al: Chronic motor cortex stimulation for the treatment of central pain. Acta Neurochir Suppl 52:137-139, 1991

Manuscript received July 17, 2001.

Accepted in final form August 9, 2001.

Address reprint requests to: Ali R. Rezai, M.D., Department of Neurosurgery, Cleveland Clinic Foundation, 9500 Euclid Avenue, Cleveland, Ohio 44195. email: rezaia@ ccf.org. 